\title{
IMPACT OF INTAKE BOUNDARY LAYER TURBULENCE ON THE COMBUSTION BEHAVIOR IN A SCRAMJET
}

\author{
A. Mack, J. Steelant, V. Togiti, and J. M. A. Longo
}

The present investigations focus on the mixing and combustion enhancement capabilities of a boundary layer entering a combustion chamber. Numerical investigations for both a generic configuration and a real ramp compression intake of a full-scale vehicle with a combustion chamber height of $0.3 \mathrm{~m}$ were performed. The flow condition is chosen according to possible conditions in a scramjet combustion chamber with 1300-kelvin static temperature, 1-bar static pressure, and a Mach number of 3 . The results of this sensitivity study show that both mixing and combustion efficiency are significantly increased due to the turbulence of the incoming boundary layer, whereas the required injector spacing to reach optimum mixing and combustion has to be increased. In addition to the classical porthole injection, an injection system with hypermixing capabilities was investigated which showed comparable sensitivities.

\section{INTRODUCTION}

For an air-breathing supersonic combustion ramjet (scramjet), fuel injection, mixing, and combustion play an important role for the vehicle net thrust at supersonic flight. In the past, mixing [1] and combustion [2] processes were systematically analyzed for full-scale combustion chambers at laminar flow conditions in order to maximize the mixing and combustion efficiency and, by this, reducing the required combustion chamber length. For generic ducts with homogenous undisturbed inflow, different wall injection systems and injector spacings were investigated to maximize the performance. It could be shown that the turbulent flow generated by the wall boundary layers downstream of the injector and the shear layers of the injected fuel did not have a significant influence on mixing and combustion [2].

The present investigation focuses on the impact of a turbulent boundary layer flow entering the combustion chamber such as that from an intake ramp. It was shown experimentally in the past that the presence of thick boundary layers in the range of 2-3 injector diameters at fuel penetrations of 5-6 diameters

This is an Open Access article distributed under the terms of the Creative Commons Attribution-Noncommercial License 3.0, which permits unrestricted use, distribution, and reproduction in any noncommercial medium, provided the original work is properly cited. 
improves the mixing strongly $[3,4]$. For full-scale vehicles such as the LAPCAT M-8 configuration [5], the length of the intake ramp is of the order of $40 \mathrm{~m}$; due to this, a very thick boundary layer enters the combustion chamber. To increase the thrust efficiency on system level, a boundary layer bleed should be avoided. By this, the fully turbulent boundary layer enters the duct. First numerical investigations have shown that the turbulent boundary layer flow interacting with the injected hydrogen fuel increases the mixing process drastically. This shows a larger impact than the previously investigated parameters in the generic ducts of [1] and [2].

\section{GENERIC CONFIGURATION}

The combustion chamber dimensions are related to the full-scale combustion chamber of a future supersonic vehicle. The height of the combustion chamber is fixed at $h_{\mathrm{cc}}=0.3 \mathrm{~m}$. Following the strategy with wall injection from both upper and lower combustion chamber walls, the computational domain employing symmetry conditions is reduced to $h=0.15 \mathrm{~m}$. The width depends on the injector spacing $s=2 w$ (Fig. 1). The symmetry planes allow to reduce the computational effort. Of course, in reality, small disturbances would lead to changes in the shock patterns and the flowfield which is not considered here. The ducts have dimensionless cross section of $W \times H=5 \times 5,5 \times 7.5,5 \times 10$, and $5 \times 20$ (cf. also Fig. 1). The dimensionless injector diameter $D=d / w=1 / 5$ is kept constant for all cases. The length of all combustion chambers is $l=1.8 \mathrm{~m}$, i.e., the dimensionless length is $L=l / h=12$.

In the previous investigations, an undisturbed parallel inflow entered the duct. In the present study, a generic boundary layer was created originating

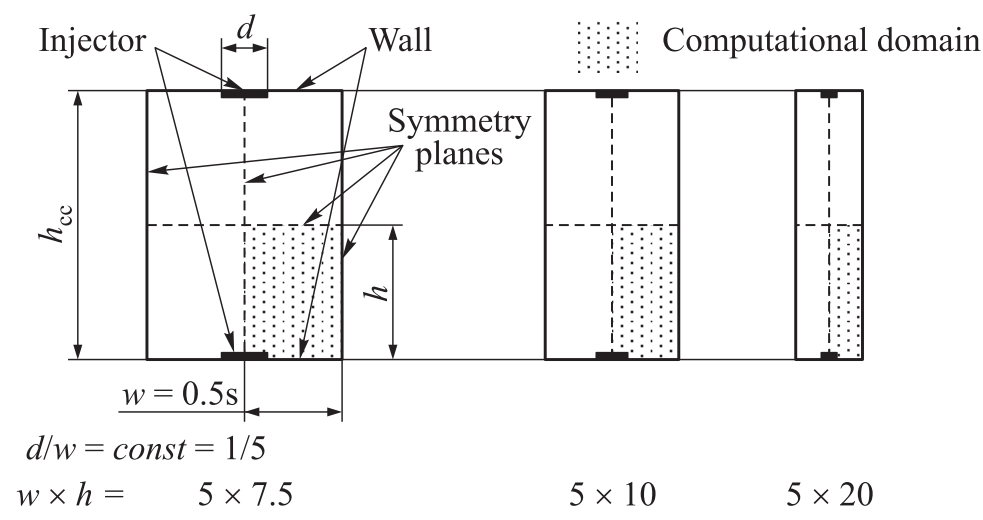

Figure 1 Duct cross sections and computational domain 


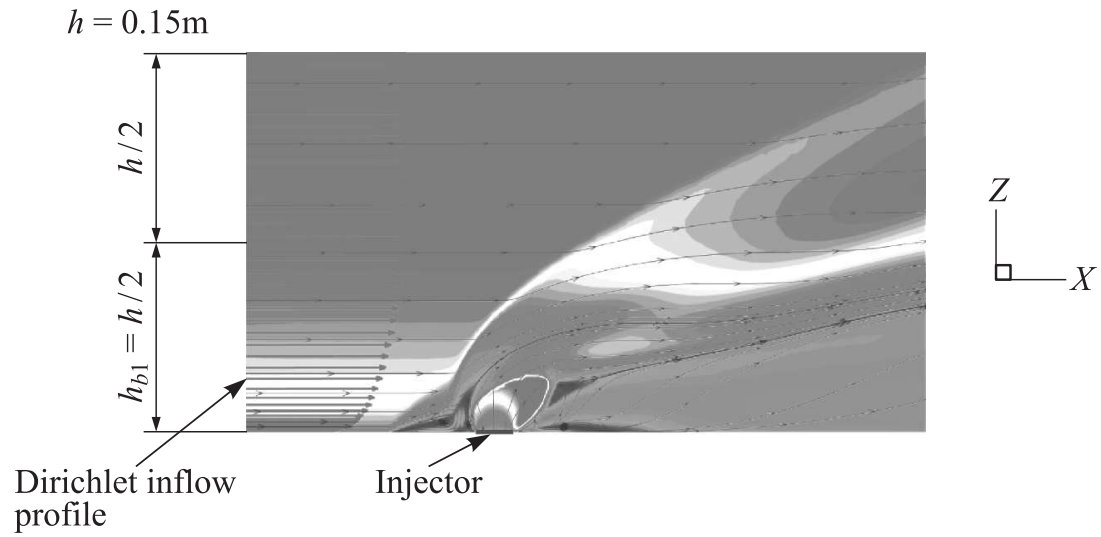

Figure 2 Boundary layer inflow profile (Mach number contours)

Table 1 Flow conditions in the generic duct

\begin{tabular}{lccccc}
\hline \multicolumn{1}{c}{ Flow } & $\mathrm{M}$ & $p, \mathrm{kPa}$ & $p_{t}, \mathrm{bar}$ & $T, \mathrm{~K}$ & $\rho, \mathrm{kg} / \mathrm{m}^{3}$ \\
\hline Air & 3 & 100 & 39.63 & 1300 & 0.2694 \\
$\mathrm{H}_{2}(5 \times 7.5)^{a}$ & 1 & $1,402.160$ & 26.54 & 250 & 1.360 \\
$\mathrm{H}_{2}(5 \times 10)^{a}$ & 1 & $1,870.234$ & 35.40 & 250 & 1.814 \\
$\mathrm{H}_{2}(5 \times 20)^{a}$ & 1 & $4,686.926$ & 88.72 & 250 & 4.546 \\
\hline${ }^{a}$ For $\Phi=1.0$ & & & & &
\end{tabular}

from a flat plate in front of the combustion chamber. The length of the flat plate is $6.2 \mathrm{~m}$. The boundary layer thickness $h_{\mathrm{bl}}$ has a height $0.25 h_{\mathrm{cc}}(50 \%$ of computational domain $h$, Fig. 2) which corresponds roughly to the relative boundary layer thickness of a full-scale ramp intake like proposed in [5]. The flow conditions are taken from typical combustion chambers: 3.0 for the Mach number, 1-bar static pressure, and 1300-kelvin static temperature. Detailed flow conditions are given in Table 1.

The desired equivalence ratio is 1 . The fuel injection pressure has to be correlated with the inlet area of the computational domain and depends on the injector spacing.

In order to estimate the performance of an injection system, the total pressure loss and the mixing efficiency are calculated. The air-based mixing efficiency $\eta_{m}$ at a station of interest $x$ is defined as the ratio of the mass flux of oxygen that would react after mixture ignition to the mass flux of oxygen entering the duct:

$$
\eta_{m}(x)=\int_{x} \frac{c_{\mathrm{O}_{2}}^{R}}{\dot{m}_{\mathrm{O}_{2}, x=0}} d \dot{m} .
$$


The mass fraction of reacting oxygen is:

$$
c_{\mathrm{O}_{2}}^{R}=\min \left(c_{\mathrm{O}_{2}}, \frac{c_{\mathrm{O}_{2}}^{S} c_{\mathrm{H}_{2}}}{c_{\mathrm{CH}_{2}}^{S}}\right) .
$$

The stoichiometric mass fraction of hydrogen $c_{\mathrm{H}_{2}}^{S}$ is equal to 0.02876 and the stoichiometric mass fraction of oxygen $c_{\mathrm{O}_{2}}^{S}$ is 0.22824 .

The combustion efficiency $\eta_{c}$ at a station of interest $x$ is defined as the ratio of mass flux of reacted hydrogen to the mass flux of injected hydrogen:

$$
\eta_{c}(x)=1-\int_{x} \frac{c_{\mathrm{H}_{2}}}{\dot{m}_{\mathrm{H}_{2}, \mathrm{inj}}} d \dot{m} .
$$

The total pressure loss due to viscous forces, flow separation, mixing, and shock waves is defined as:

$$
\Pi(x)=1-p_{0, \text { rec }}(x)
$$

with the pressure recovery being:

$$
p_{0, \mathrm{rec}}(x)=\frac{\int_{x} \rho u p_{0} d A}{\int_{x=0} \rho u p_{0} d A} .
$$

\section{NUMERICAL MODELING}

The computational fluid dynamics (CFD) solutions are achieved with the unstructured DLR TAU-Code that has been validated in the past for different configurations at super- and hypersonic flow conditions [6], including extensive studies of wall jet injection [7,8] and combustion [9]. The time-accurate three-dimensional Navier-Stokes equations are marched for steady or unsteady conditions by an explicit three-stage Runge-Kutta scheme or an implicit LowerUpper Symmetric Gauss-Seidel (LUSGS) scheme. Different standard upwind solvers such as Van-Leer, advection upstream splitting method (AUSM), and AUSMDV and gas modeling formulations for perfect gas, thermochemical equilibrium and nonequilibrium are available. State-of-the-art computation acceleration techniques such as local time stepping, residual smoothing, and multigrid are implemented. A variety of one- and two-equation turbulence models including detached-eddy simulation (DES) and large-eddy simulation (LES) can be employed. 
All computations are performed with fully turbulent wall boundary conditions employing the Spalart-Allmaras (SA) or $k-\omega$ turbulence model. The wall temperature is set to $1000 \mathrm{~K}$. No turbulence combustion coupling was employed so far. As investigations in [10] have shown, there is no significant enhancement of the combustion process due to turbulence in the configurations like the present one.

The mixing calculations have been performed using a nonreacting mixture model for two perfect gases. Hydrogen and air are modeled as perfect gases employing two species equations. The diffusion is modeled by Fick's law with a Schmidt-number of 0.7.

The Evans-Schexnayder 7-species 8-reaction set [11] is taken as combustion modeling for the chemical nonequilibrium flow calculations including combustion. The forward reaction rates are described by the Arrhenius law, whereas the backward rates are calculated using the equilibrium constants.

The hybrid grids are generated with 32 prism cells in the boundary layer and are locally adapted several times such that the overall number of grid points is between 150,000 (initial grid) and $1.0 \cdot 10^{6}-1.8 \cdot 10^{6}$ (final grid).

The flat-plate boundary layer flow entering the duct was treated as twodimensional (2D) in order to reduce the numerical effort. The 2D solution was staggered in spanwise direction and applied as a Dirichlet boundary condition at the duct inflow plane.

The flow conditions were identical with the previous cases, see Table 1.

The ramp intake was operated under conditions of the LAPCAT* vehicle at cruise flight $(\mathrm{M}=8)$ and also applied at the combustion chamber intake plane as boundary conditions.

\section{RESULTS}

\subsection{Mixing Results with Generic Boundary Layer Inflow}

Pure mixing calculations were performed for hydrogen injected into the ducts of dimensionless cross-section areas of $5 \times 7.5,5 \times 10$, and $5 \times 20$ (Fig. 3 ) and equivalence ratios between 1.0 and 0.5 . Due to the boundary layer, the air mass flow in the duct is reduced compared with the results from $[1,2]$ by $10.5 \%$.

The turbulence of the incoming boundary layer influences the flow topology which reduces the penetration and damps the vortex generated by the injection process. The mixing of hydrogen with air after exiting the injector is enhanced: hydrogen is consumed before penetrating deeper into the duct. Due to this, the bow shock in front of the injector is reflected further down compared with the previous results without the boundary layer entering the duct. In the previous

\footnotetext{
*LAPCAT — Long-term Advanced Propulsion Concepts And Technologies.
} 


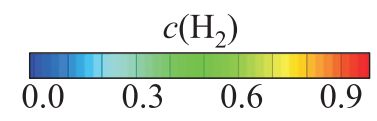

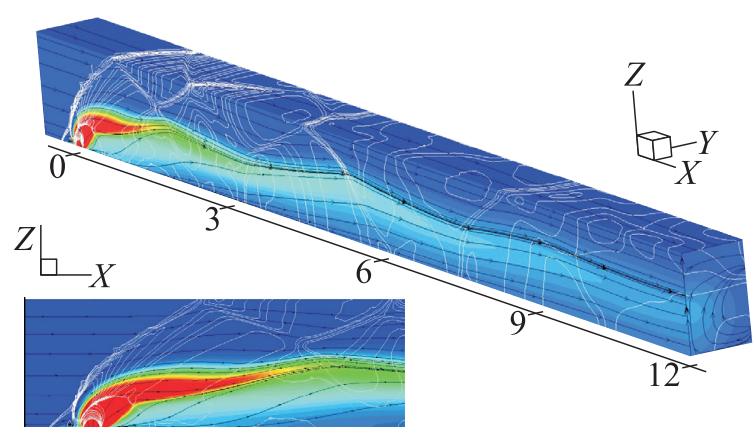

(a)

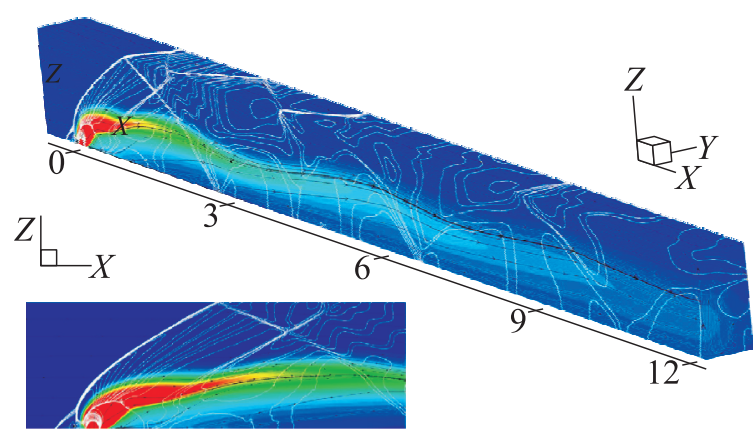

(b)

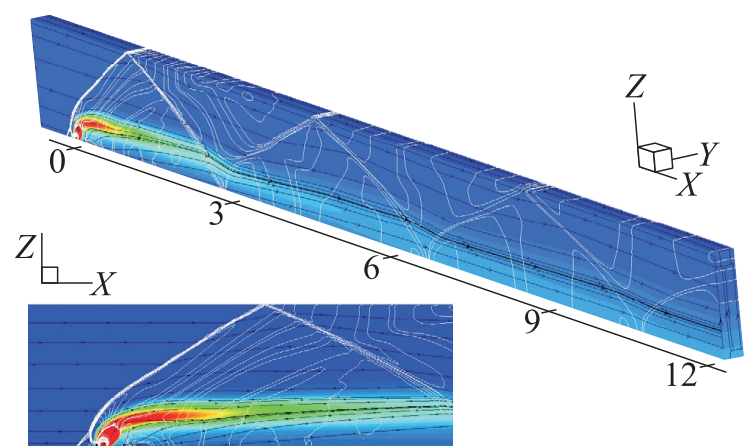

(c)

Figure 3 Mixing results for ducts with aspect ratios of $5 \times 7.5(a), 5 \times 10(b)$, and $5 \times 20(c)$. (Refer Mack et al., p. 536.) 
results, the laterally reflected shocks led to a significant increase in penetration due to the redirection of the flow crossing the shocks such that the injected hydrogen reached the upper symmetry plane. The present cases cannot take much advantage of this effect: the hydrogen stays mainly in the lower part of the duct $(5 \times 7.5$ and $5 \times 10)$. With the $5 \times 20$ duct, the penetration is very low and is further reduced by the bow shock reflected from the upper symmetry plane and the wall. The shock pattern is almost 2D; the lateral reflections are very weak and damped in the viscous boundary layer. The mixing takes place within the incoming boundary layer; therefore, the vortical flow generated by the injection is strongly weakened compared with the previous results [1]. Nevertheless, the presence of turbulence in the main flow increases the mixing significantly which can be seen in Figs. 4 to $6(\Phi=1.0)$. Figure 7 compares the present results with the results of previous investigations. The mixing efficiency of the $5 \times 7.5$ duct is $61 \%$. The $5 \times 10$ duct reaches a mixing efficiency of $66 \%$ which for both ducts is a factor of more than 2 higher as compared with [1], see Fig. 7 . The $5 \times 20$ duct reaches $52 \%$ mixing efficiency which is only slightly increased. Due to these results, the optimum duct cross section which defines the injector spacing is a $5 \times 10$ or even $5 \times 7.5$ ratio rather than the $5 \times 20$ as stated in [1]. With high duct aspect ratios, penetration is too low and the rotating vortex cannot be established and is damped strongly in the turbulent boundary layer flow. By this, the hydrogen flow close to the wall is separated from the air flow in the upper part of the duct. Large-scale mixing does not take place; the positive effect of the presence of the small-scale mixing in the turbulent region vanishes. For the three duct cross sections, a variation of the equivalence ratio from $\Phi=1.0$, 0.7 , and 0.5 was investigated. Mixing efficiencies increase with decreasing the equivalence ratio, although the penetration is reduced due to the lower injection pressure and reduced hydrogen mass flow. A comparable behavior for all duct sizes is observed. The relative increase in the mixing efficiency $(\Phi=0.7)$ is highest for the $5 \times 20$ duct $(27 \%)$, whereas for the $5 \times 10$ and $5 \times 7.5$ ducts, it attains $13 \%$ and $10 \%$, respectively. Nevertheless, the maximum mixing efficiency is reached for the $5 \times 10$ duct $(75 \%)$.

For the $5 \times 20$ duct, a tilted injection at $60^{\circ}$ was investigated which showed the same behavior as in [1]. Due to better penetration, the mixing efficiency is increased up to $x / h=5$, further downstream, the improvement is slightly reduced. Mixing is increased from $51 \%$ to $56 \%$. The generated vortex is weaker because the inclined injection imposes less distortion to the main flow. Due to this, the total pressure loss is reduced by $25 \%$. For the $5 \times 7.5$ duct, the total pressure loss is $23 \%$ and $27 \%$ for the $5 \times 20$ duct. The pressure losses are strongly linked to the overall mixing efficiency.

In order to keep the results as general as possible, the tilted injector was not considered further on. Nevertheless, all improvements discussed in [1,2] such as injection angle, injector shape and design, as well as injection Mach number seem to be valid also for the present flow conditions. For a real application, a 


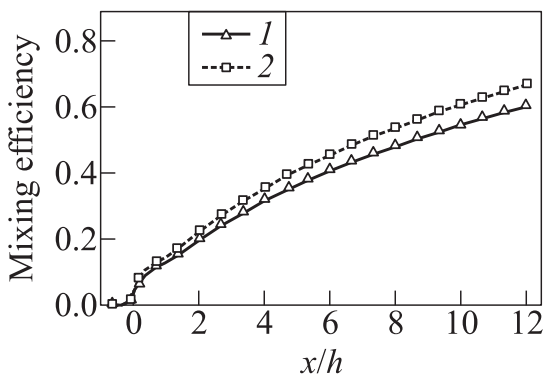

(a)

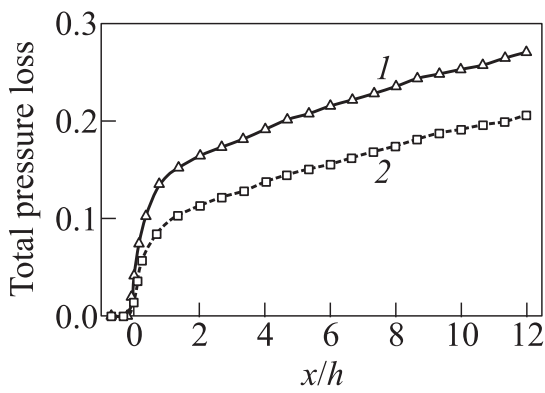

(b)

Figure 4 Mixing efficiency $(a)$ and total pressure loss $(b)$ for $5 \times 7.5$ duct at different equivalence ratios: $1-\Phi=1.0$ and $2-\Phi=0.7$

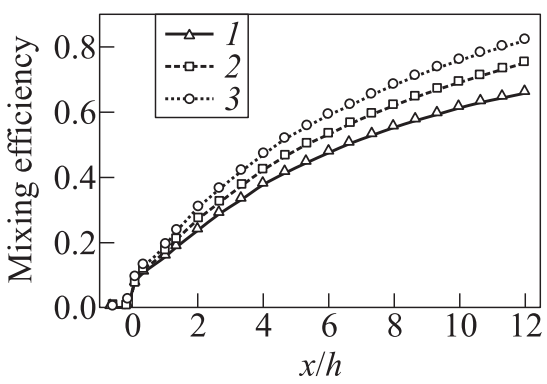

(a)

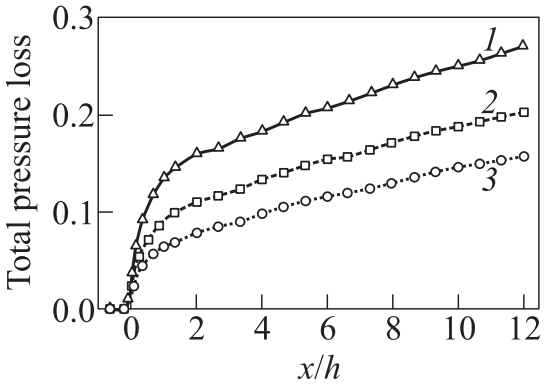

(b)

Figure 5 Mixing efficiency $(a)$ and total pressure loss $(b)$ for $5 \times 10$ duct; variation of equivalence ratio: $1-\Phi=1.0 ; 2-0.7$; and $3-\Phi=0.5$

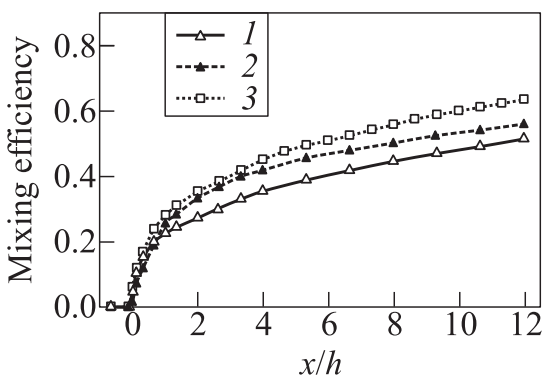

(a)

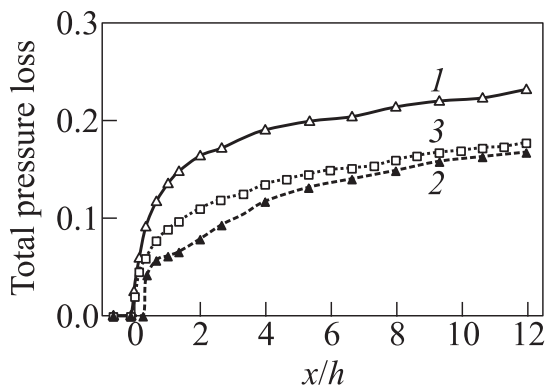

(b)

Figure 6 Mixing efficiency $(a)$ and total pressure loss $(b)$ for $5 \times 20$ duct; variation of equivalence ratio: $1-\Phi=1.0 ; 2-\Phi=1.0, \psi=60^{\circ} ; 3-\Phi=0.7$ 


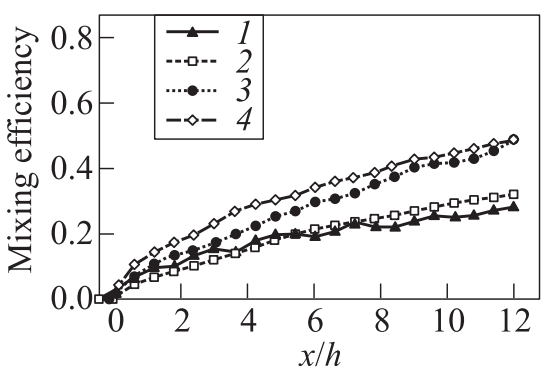

(a)

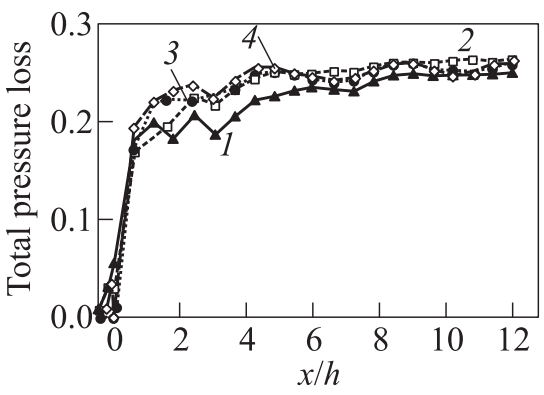

(b)

Figure 7 Mixing efficiency $(a)$ and total pressure loss $(b)$ without incoming boundary layer [1] for ducts with aspect ratios of $5 \times 5(1) ; 5 \times 10(2) ; 5 \times 20(3)$; and $5 \times 30$ (4)

tilted injection should also be chosen due to a better overall net thrust on system level.

\subsection{Combustion Results with Generic Boundary-Layer Inflow}

Including the combustion process changes the flow topology significantly depending on the equivalence ratio. Due to the improved mixing, the heat release and pressure rise during combustion leads to separation in the combustion chamber (Fig. 8). With an equivalence ratio of $\Phi=1.0$, thermal choking with a normal shock in front of the injector occurs for all duct cross-section sizes. For better clarity, the Mach number is shown for this case. At $\Phi=0.7$, a stable combustion process including a large subsonic reverse flow region behind the injector is observed. This zone is trapped inside the bow shock and its first reflection at the upper symmetry plane. The combustion takes place in this subsonic region (injector plane) behind the injector but also further downstream the duct where the flow is again accelerated to supersonic speed.

The mixing efficiencies and total pressure losses are shown in Fig. 9 to 11. For the partially subsonic combustion at $\Phi=0.7$, the combustion efficiencies reach $76 \%(5 \times 7.5), 87 \%(5 \times 10)$ and $66 \%(5 \times 20)$. Thus, the $5 \times 10$ duct shows the highest combustion efficiency at a total pressure loss of $68 \%$. This indicates that high combustion efficiencies are obtained at high losses in total pressure which have to be compensated on system level.

Only the case with $\Phi=0.5(5 \times 10$ duct, see Fig. 10) shows a flow topology which is almost identical to the pure mixing solution. Due to the supersonic combustion process, the averaged pressure increases linearly in the duct. As a result, the shock impingements enhancing the mixing process are not that strong 


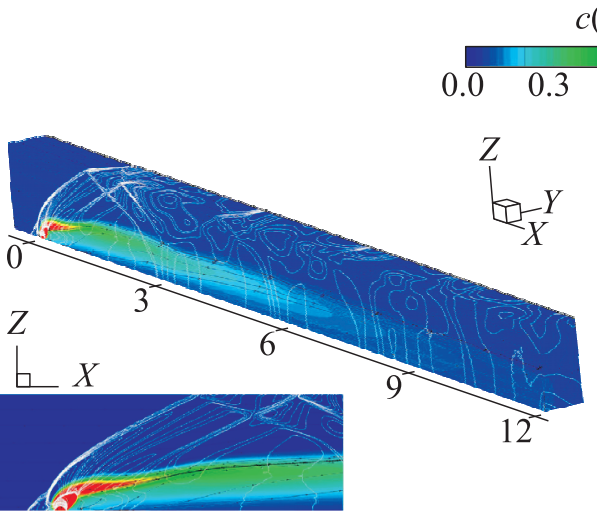

(a)
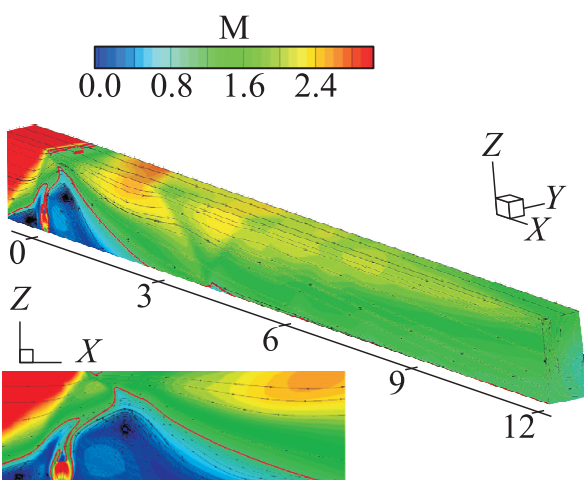

(c)

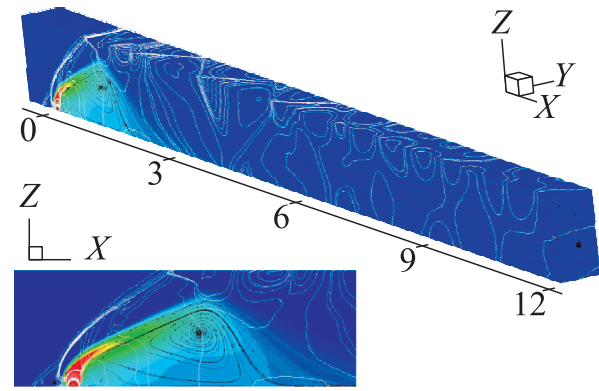

(b)
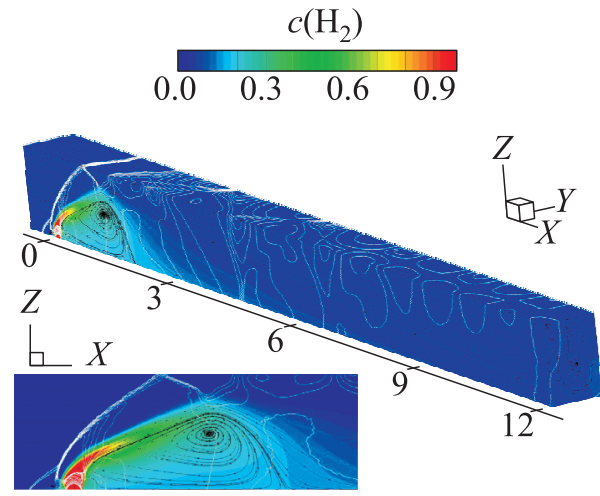

$(d)$

Figure 8 Combustion results for ducts with aspect ratios of $5 \times 10(\Phi=0.5(a)$, $0.7(b)$, and $\Phi=1.0(c))$ and $5 \times 7.5(\Phi=0.7)(d)$. (Refer Mack et al., p. 540.)

any more. The combustion efficiency reaches $78 \%$ (at pure mixing efficiency of $82 \%$, see Fig. 10). The total pressure loss is $43 \%$.

\subsection{Sensitivity of Numerical Modeling}

The numerical modeling concerning turbulence and diffusion was investigated in order to give quantities of possible variations. In Fig. 12, the results for the one-equation SA and the 2-equation $k-\omega$ turbulence models are shown. The simulation includes the full flow domain. The $2 \mathrm{D}$ inflow calculations were also performed with the corresponding turbulence model. For both equivalence ratios, 


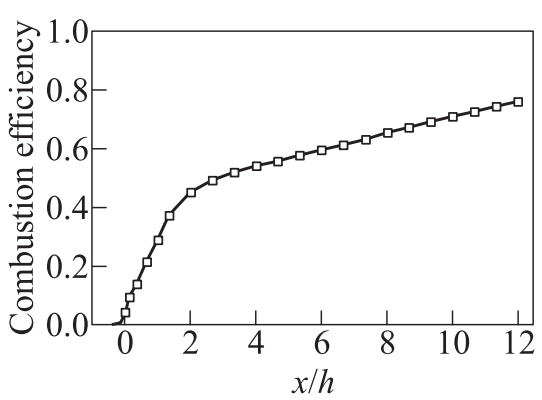

(a)

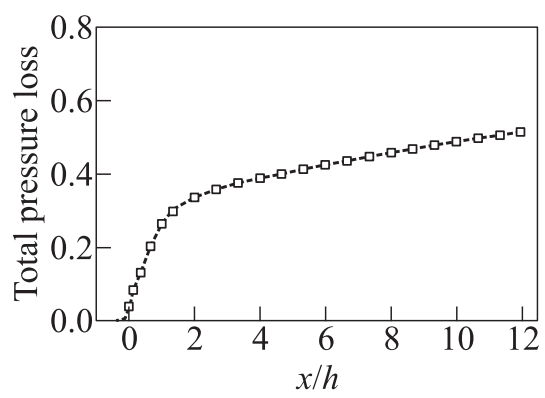

(b)

Figure 9 Combustion efficiency $(a)$ and total pressure loss $(b)$ for $5 \times 7.5$ duct at $\Phi=0.7$

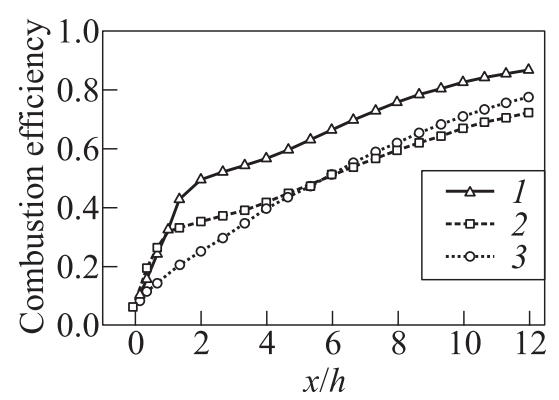

(a)

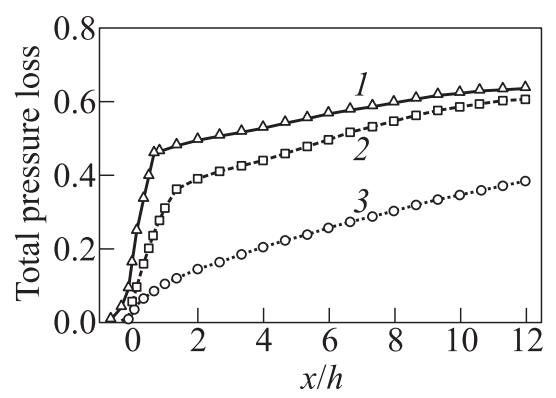

(b)

Figure 10 Combustion efficiency $(a)$ and total pressure loss $(b)$ for $5 \times 10$ duct; variation of equivalence ratio: $1-\Phi=1.0 ; 2-\Phi=0.7$; and $3-\Phi=0.5$

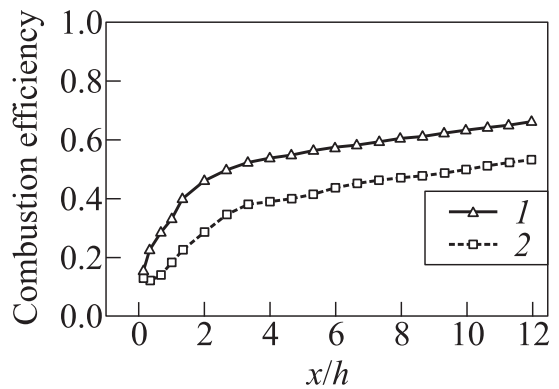

(a)

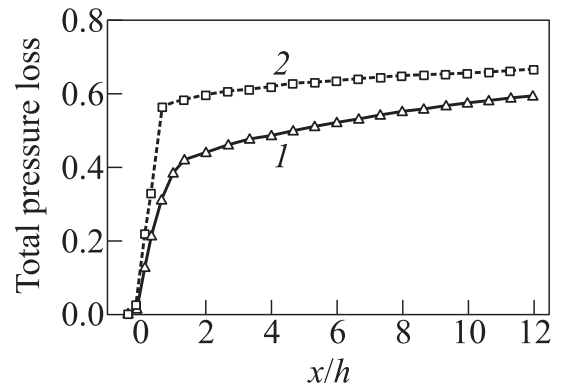

(b)

Figure 11 Combustion efficiency $(a)$ and total pressure loss $(b)$ for $5 \times 20$ duct; variation of equivalence ratio: $1-\Phi=1.0$ and $2-\Phi=0.7$ 


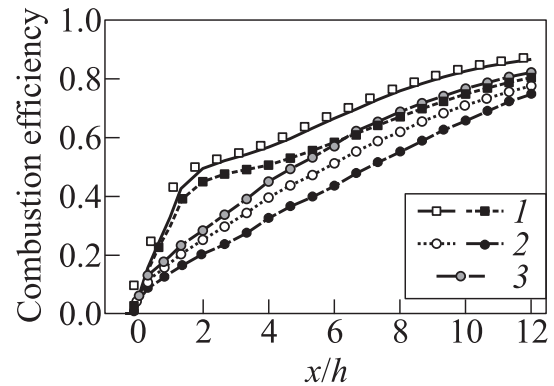

(a)

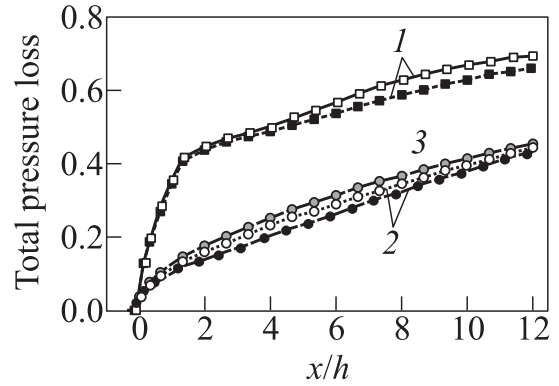

(b)

Figure 12 Combustion efficiency $(a)$ and total pressure loss $(b)$ for $5 \times 10$ duct; variation of numerical models (empty signs refer to SA model, and black signs refer to $k-\omega$ model) and equivalence ratio: $1-\Phi=0.7 ; 2-\Phi=0.5$; and $3-\Phi=0.5$, $\mathrm{Sc}=0.5$

0.7 and 0.5 , the 2-equation solution gives slightly lower values for the equivalence ratio. The mass flow rate of oxygen at the Dirichlet inflow plane of the combustion chamber calculated with the 2-equation turbulence model is $1.5 \%$ lower compared with the 1-equation model. Differences of $4 \%$ to $7.5 \%$ in the combustion efficiencies occur at the duct exit plane. Larger differences occur at higher equivalence ratio $(0.7)$, since the combustion process takes place mainly in the subsonic reverse flow regime where turbulence-combustion coupling might play a larger role. Without spatially abundant presence of boundary-layer generated turbulence entering the duct, the influence of the turbulence was negligible independently from the turbulence model [2]. The combustion efficiencies were in the range of $30 \%-55 \%(5 \times 5-5 \times 20$ duct, $\Phi=0.5$ to 1$)$. On the contrary, the present investigations with the boundary-layer flow entering the duct show much higher combustion efficiencies of $54 \%-87 \%$ with a sensitivity to the turbulence model of only $4 \%-7.5 \%$. This efficiency increase is therefore mainly dependent on the turbulent flow entering the duct rather than on the applied turbulence model in the mixing and combustion area.

The diffusion of the different gases is modeled according to Fick's law via the Schmidt number. A default value of $\mathrm{Sc}=0.7$ was taken in the present and all previous studies. An additional calculation with enhanced diffusion at $\mathrm{Sc}=0.5$ was performed. The combustion efficiency is increased by roughly $5 \%$ due to the improved diffusion of the species.

Due to the combustion process, the static pressure in the combustion chamber increases. In Fig. 13, the static surface pressure in the spanwise middle section is shown. As expected from the flow topology, the $\Phi=0.5$ solution $(5 \times 10$ duct) shows the typical monotonous averaged pressure increase at supersonic 
combustion in the duct. The impingement of the reflected injection bow shock is clearly visible at $x / h=3.5$, $6.5,8.5$, and 11 . The other cases with the locked subsonic reverse flow region behind the injector (cf. Fig. 8) show a large pressure increase behind the injector until the impingement of the reflected bow shock at $x / h=4$. Further downstream, the flow accelerates again to supersonic speed and the averaged pressure increases monotonically to an exit pressure of 2.9 to 3.3 bar similar to the flow at $\Phi$ $=0.5$. The supersonic combustion process at $\Phi=0.5$ provides a pressure of 2.5 bar. The higher combustion efficiencies and static pressures in the combustion chamber are obtained with much higher losses in total pressure. Due to the subsonic reverse flow region, the total pressure loss increases from $45 \%$ to almost $70 \%$. Whether the higher combustion efficiencies can compensate the larger pressure losses has to be traded off on system level. An interesting aspect is that between a smooth supersonic combustion and the thermal choking of the duct, a stable flowfield with partially subsonic combustion can be established. Due to the local pressure peaks caused by the reflected bow shock, the subsonic regions including separation or subsonic pockets can be trapped between the reflections. This was also shown in [2] where a subsonic pocket was trapped at the end of the combustor between the shock reflections. Even the semiempirical Korkegi correlation [12] seems to predict the subsonic zones; it gives a certain maximum pressure increase at a corresponding Mach number before separation occurs. The Korkegi correlation reads:

$$
\frac{p_{s}}{p}=1+0.3\left(\frac{U}{a}\right)^{2} \text { for } \quad \frac{U}{a}<4.5 .
$$

The static pressures calculated by CFD are locally above the pressure predicted by the Korkegi correlation (see Fig. 13). This behavior was also observed with the HyShot investigations at equivalence ratios between 0.55 and 0.7 [13]. Subsonic reverse-flow combustion areas, originating from local pressure peaks of the reflected bow shock, showed an abrupt pressure increase in the combustion chamber. In contrary, supersonic combustion always showed an almost linear averaged pressure increase along the combustion chamber with only local peaks from the shock reflections. 


\subsection{Comment}

Of coarse, real scramjets have a better net thrust production at equivalence ratios close to one to overcome pressure losses distinctibly. Thermal choking as it appears here at an equivalence ratio of one or in large subsonic reverse-flow combustion areas occurring at an equivalence ratio of 0.7 may not take place. For a real application, a different duct shape (e.g., diverging duct) or an adapted flow condition (e.g., higher duct flow Mach number) has to be chosen to avoid thermal choking or separation due to large pressure rise. Due to the extensive work being done so far for this simple generic duct, no changes were done to the geometry to keep the configuration as generic as possible and to preserve comparability. Optimizing the duct geometry and/or flow conditions might be done in future work.

\subsection{Mixing Investigations Including Ramp Intake Compression}

The mixing behavior in the combustion chamber of a realistically configured vehicle including the forebody intake ramp flow was investigated at cruise flight conditions (Table 2). The geometry is a preliminary intake from the LAPCAT M-8 vehicle with the ramps of approximately $40 \mathrm{~m}$ long, and theoretical onedimensional (1D) compression ratio from 3-external and 2-internal ramps of 178 . No boundary layer bleed is foreseen due to performance issues, the turbulent boundary layer is swallowed completely by the combustion chamber (Fig. 14). Again, fuel injection from both sides is foreseen. Wall porthole injectors $(\mathrm{M}=1$, $\psi=90^{\circ}$ ) are compared with the Hypermixer geometry [14] (cf. also Fig. 15). In the Hypermixer, the fuel is injected from a 12 degree ramp at an angle of 12-degree, a backward facing ramp increases the duct height from 0.3 to $0.376 \mathrm{~m}$ at the injector position. Due to this, a strong vortex is created in which the fuel is injected. The Hypermixer geometry was scaled up from the HyShot experiment to a duct height of $0.3 \mathrm{~m}$ as for all ducts in the present paper. For

Table 2 Flow condition in ramp intake (LAPCAT M8 vehicle)

\begin{tabular}{llcccc}
\hline \multicolumn{1}{c}{ Flow } & $\mathrm{M}$ & $p, \mathrm{kPa}$ & $p_{t}, \mathrm{bar}$ & $T, \mathrm{~K}$ & $\rho, \mathrm{kg} / \mathrm{m}^{3}$ \\
\hline Free stream $^{a}$ & 8.03 & 0.6329 & 63.32 & 234.6 & 0.0094 \\
Duct & 2.5 & 170 & 28.50 & 1450 & 0.405 \\
$\mathrm{H}_{2}(5 \times 7.5)^{b}$ & 1 & 1210.320 & 22.912 & 242 & 1.215 \\
$\mathrm{H}_{2}(5 \times 20)^{b}$ & 1 & 4035.200 & 76.388 & 242 & 4.05 \\
\hline
\end{tabular}

${ }^{a}$ Altitude $34 \mathrm{~km}$.

${ }^{b}$ For $\Phi=1.0$ 


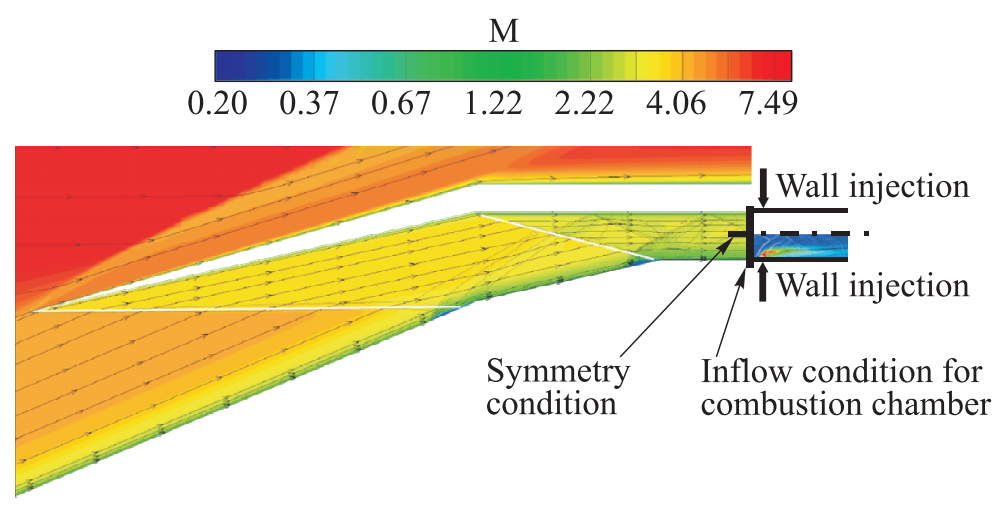

Figure 14 Intake and combustion chamber geometry of LAPCAT M8 vehicle (without boundary layer bleed): at $\mathrm{M}=8 ; T_{\text {wall }}=1000 \mathrm{~K}$ (pressure isoliness). (Refer Mack et al., p. 545.)

better comparison, the injectors are both located at $x=0$. The ducts have a dimensionless cross section of $5 \times 7.5$, the Hypermixer duct due to its area increase has a dimensionless cross section of $5 \times 8.33$ (inflow plane) to $5 \times 10.4$ (behind the injector). In contrary to the generic results available so far (see Fig. 2), the boundary layer thickness is roughly $75 \%$ of the duct height according to the compression ramp flow. Due to computational requirements, only the lower half of the combustion chamber was modeled. The upper boundary of the computational domain was modeled as the symmetry plane (see Fig. 14). The intake calculations were performed in $2 \mathrm{D}$ and interpolated on the inflow plane of the combustion-chamber inflow boundary. The flow topologies of the two injection systems are shown in Fig. 15. The penetration of the porthole injection is slightly less than in the cases studied previously (cf. Fig. 3), due to the thicker boundary layer and impinging shocks from the ramp flow the turbulent viscosity is higher. This increases the mixing and much more of the fuel is consumed and mixed instead of penetrating unmixed into the duct. The generated vortex is less strong than with the laminar cases or the ones with the generic boundary layer. The Hypermixer creates a strong vortex due to the wall geometry and not due to the injection process. Therefore, the total pressure loss is higher for the Hypermixer. Up to the injection plane, $10 \%$ of total pressure loss is accumulated due to the geometrical shape. At the combustion chamber exit plane, $33 \%$ is reached (Fig. 16). The wall porthole injection provides only $28 \%$ $(5 \times 7.5$ duct $)$.

Due to the strong vortex created by the Hypermixer, at $x / h>2$, the mixing efficiency is higher compared with the wall injectors. It reaches $76 \%$ at the duct 


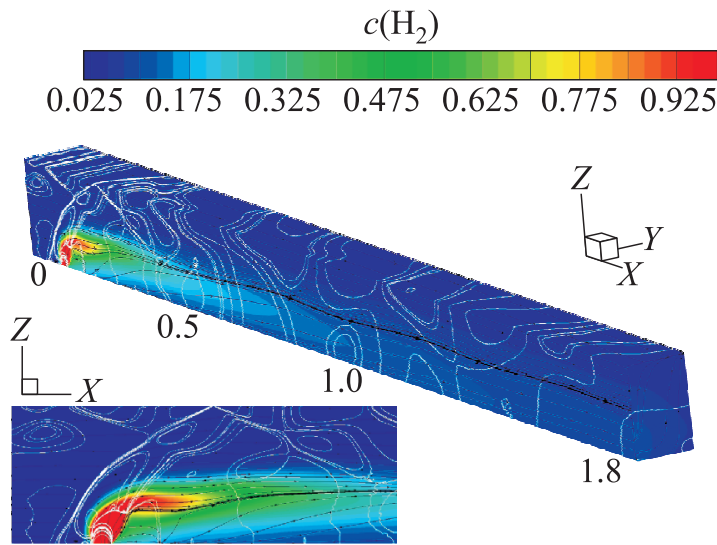

(a)

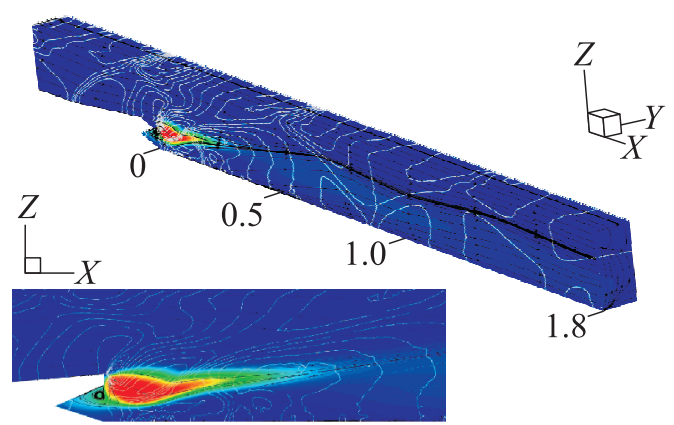

(b)

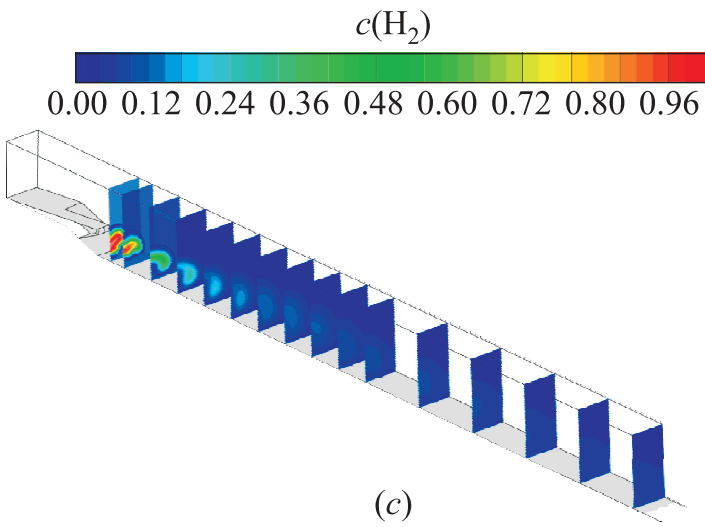

Figure 15 Mixing results for porthole injection $(a)$ and Hypermixer ( $b$ and $c$ ), $\Phi=1.0$. (Refer Mack et al., p. 546.) 


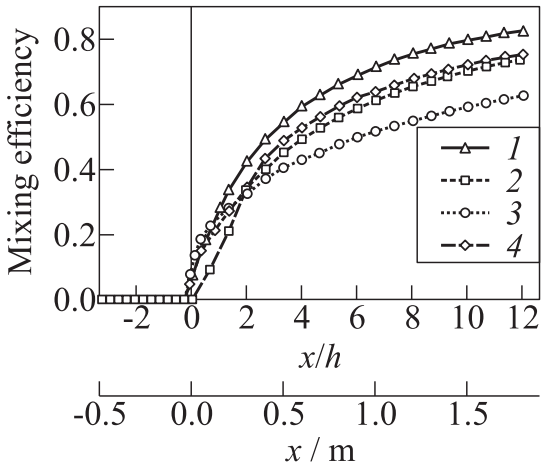

(a)

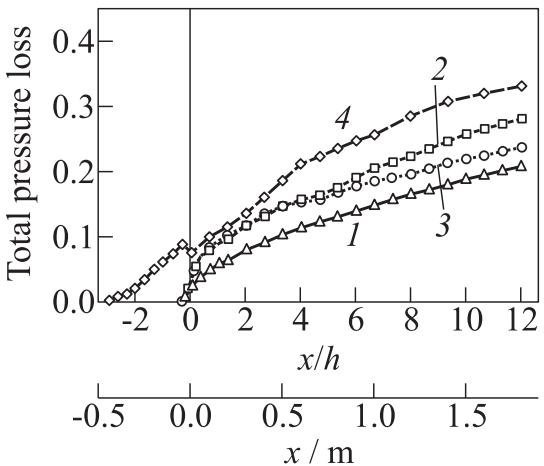

(b)

Figure 16 Mixing efficiency $(a)$ and total pressure loss $(b)$ for $5 \times 7.5(1-\Phi=0.5$ and $2-\Phi=1.0)$ and $5 \times 20(3-\Phi=1.0)$ ducts and Hypermixer $(4-\Phi=1.0)$

exit plane (wall injector: $73 \%$ in $5 \times 7.5$ duct). The gradient of mixing for both injector types is almost identical at $x / h>8$. According to this, an extrapolation until 100 percent mixing efficiency would give a slightly shorter required duct length for the Hypermixer (see Fig. 16).

If the required length upstream the injector is taken into account for both Hypermixer and wall injector, the situation changes. The Hypermixer requires in its current design an axial length of almost $0.5 \mathrm{~m}(\Delta x / h=3.33)$ between the duct entrance and the injector plane; the wall injector only needs some space to lock its bow shock separation $(0.03 \mathrm{~m}, \Delta x / h=0.2)$.

The $5 \times 20$ duct fails to generate a strong mixing vortex as explained before for the generic cases. The mixing efficiency is much lower and reaches $62 \%$.

Decreasing of the equivalence ratio does not affect the vortex generation with the Hypermixer configuration. The vortex is created by the surfaces of the combustion chamber rather than by the injected fuel. Figure 17 shows the mixing behavior for ducts of a length of $2.6 \mathrm{~m}(x / h=17.33)$, the previously considered duct length of $1.8 \mathrm{~m}(x / h=12)$ is indicated by the vertical dashed line. With an equivalence ratio of 0.5 , even after $x / h=12$, full mixing is achieved.

The porthole injector only reaches $83 \%$ mixing efficiency due to its small penetration at lower equivalence ratio (see Fig. 16). The total pressure loss, in contrary to the wall porthole injectors, decreases with increasing the equivalence ratio. The injected fuel fills the dead water zones and reduces the reverse flow region behind the injector.

The mixing efficiency for an undisturbed laminar inflow in the duct is also shown in Fig. 17. Due to the vortex, which is actively generated by the injector, the mixing efficiency is quite high for this case $(61 \%$ at $x / h=12)$ compared with the wall porthole injectors (roughly $30 \%$, see Fig. 7 ). 


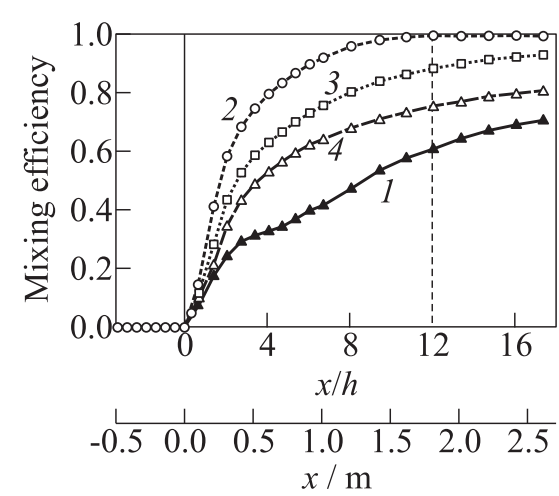

(a)

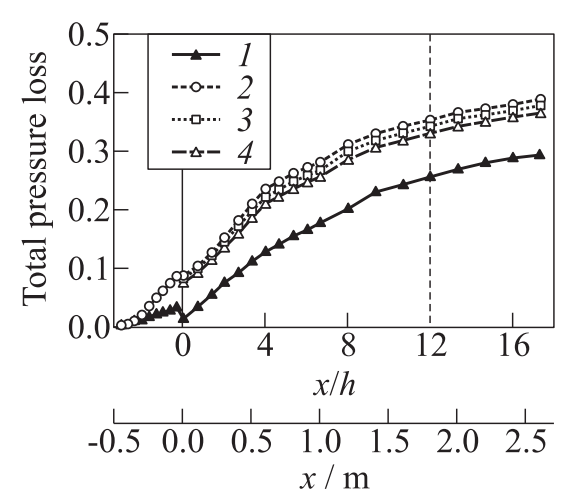

(b)

Figure 17 Mixing efficiency $(a)$ and total pressure loss $(b)$ for Hypermixer; variation of equivalence ratio: $1-\Phi=1.0$ (free stream) $; 2-\Phi=0.5 ; 3-\Phi=0.75$; and $4-$ $\Phi=1.0$

The Hypermixer has always the disadvantage of higher total pressure losses. However, especially in low-turbulence flow or throttled engine operation conditions, the mixing behavior is better compared with the wall-porthole injectors at $\psi=90^{\circ}$. As shown in previous papers, the mixing process of the wall injectors can be enhanced and the total pressure loss can be reduced by supersonic injection $[1,2]$. Since the configurations were kept simple, this was not investigated here but should be done in the future.

\section{SUMMARY}

A required scramjet combustion-chamber length for full combustion is mainly dependent on the mixing and combustion behavior of the injected fuel. Based on previous CFD investigations, the main driving mechanisms for good mixing could be identified for a generic combustion chamber (length $1.8 \mathrm{~m}$, height $0.3 \mathrm{~m}$ ). For combustion chambers without any turbulent flow entering the duct, mixing and combustion are quite poor $(30 \%-55 \%)$. On the contrary, the present investigations with boundary layer flow entering the duct show much higher combustion efficiencies of $54 \%-87 \%$ with a sensitivity to the implemented turbulence model of only $4 \%-7.5 \%$. This efficiency increase is, therefore, mainly dependent on the turbulent flow entering the duct rather than on the applied turbulence model in the mixing and combustion area. For the present flow condition and geometry, an equivalence ratio of 0.5 leads to a flowfield with mainly supersonic combustion. At an equivalence ratio of 1.0, thermal choking of the duct occurs. At 
an equivalence ratio of 0.7 , a stable subsonic/supersonic mode occurs with very high combustion efficiencies but also high losses in total pressure. Due to the presence of a turbulent boundary layer, the injector spacing has to be increased to achieve the best performance. A duct cross section of $5 \times 10$ was found to be optimal.

\section{ACKNOWLEDGMENTS}

The numerical work was performed within the 'Long-Term Advanced Propulsion Concepts and Technologies' project investigating high-speed airbreathing propulsion. LAPCAT, coordinated by ESA-ESTEC, is supported by the EU within the 6th Framework Programme Priority 1.4, Aeronautic and Space, Contract no.: AST4-CT-2005-012282. Further info on LAPCAT can be found on http://www.esa.int/techresources/lapcat. ESA-ESTEC is acknowledged for the internal research fellowship granted enabling the present research work.

\section{REFERENCES}

1. Mack, A., and J. Steelant. 2006. Mixing enhancement by shock impingement in a generic scramjet combustion chamber. European Conference on Computational Fluid Dynamics ECCOMAS CFD 2006. Egmond aan Zee, The Netherlands.

2. Mack, A., J. Steelant, K. Hannemann, S. Karl, and J. Odam. 2006. Mixing and combustion enhancement in a generic scramjet combustion chamber. 14th AIAA/AHI Spaceplanes \& Hypersonic Systems \& Technologies Conference. Canberra.

3. Orth, R. C., J. A. Schetz, and F. S. Billig. 1969. The interaction and penetration of gaseous jets in supersonic flow. NASA CR-1386.

4. Rogers, C. 1971. A study of the mixing of hydrogen injected normal to a supersonic airstream. NASA Technical Note.

5. Sippel, M., and J. Klevanski. 2006. Preliminary definition of the supersonic and hypersonic airliner configurations. AIAA Paper No. 2006-7984.

6. Mack, A., and V. Hannemann. 2002. Validation of the unstructured DLR-TAUCode for hypersonic flows. AIAA Paper No. 2002-3111.

7. Kovar, A., and E. Schülein. 2004. Comparison of experimental and numerical investigations on the side jets in a supersonic cross flow. London, Great Britain: Royal Aeronautical Society.

8. Adeli, R., J. M. A. Longo, and H. Emunds. 2006. Flow field study of a supersonic jet exiting into a supersonic stream. Notes on Numerical Fluid Mechanics and Multidisciplinary Design. Vol. 92. Springer 2006: New results in Numerical and Experimental Fluid Mechanics V. 160-67. 
9. Karl, S., and K. Hannemann. 2006. Application of the DLR TAU-code to the RCM-1 testcase: Penn State Preburner Combustor. 3rd Workshop (International) on Rocket Combustion Modeling Proceedings.

10. Karl, S., K. Hannemann, J. Steelant, and A. Mack. 2006. CFD analysis of the HyShot supersonic combustion flight experiment configuration. AIAA Paper No. 2006-8041.

11. Evans, J. S., and Ch. J. Schexnayder, Jr. 1980. Influence of chemical kinetics and unmixedness on burning in supersonic hydrogen flames. AIAA J. 18(2):188-93.

12. Korkegi, R. H. 1975. Comparison of shock-induced two- and three-dimensional incipient turbulent separation. AIAA J. 13(4):534-35.

13. Steelant, J., A. Mack, K. Hannemann, and A. D. Gardner. 2006. Comparison of supersonic combustion tests with shock tunnels, flight and CFD. AIAA Paper No. 2006-4684.

14. Lee, J. 1994. Numerical study of mixing in supersonic combustors with hypermixing injectors. J. Propul. Power 10(3):297-304. 